\title{
Acknowledgement to Reviewers of Computers in 2016
}

\section{Computers Editorial Office}

Published: 11 January 2017

MDPI AG, St. Alban-Anlage 66, 4052 Basel, Switzerland; computers@mdpi.com

The editors of Computers would like to express their sincere gratitude to the following reviewers for assessing manuscripts in 2016.

We greatly appreciate the contribution of expert reviewers, which is crucial to the journal's editorial process. We aim to recognize reviewer contributions through several mechanisms, of which the annual publication of reviewer names is one. Reviewers receive a voucher entitling them to a discount on their next MDPI publication and can download a certificate of recognition directly from our submission system. Additionally, reviewers can sign up to the service Publons (https://publons.com) to receive recognition. Of course, in these initiatives we are careful not to compromise reviewer confidentiality. Many reviewers see their work as a voluntary and often unseen part of their role as researchers. We are grateful to the time reviewers donate to our journals and the contribution they make.

If you are interested in becoming a reviewer for Computers, see the link at the bottom of the webpage http://www.mdpi.com/reviewers.

The following reviewed for Computers in 2016:

\begin{tabular}{lll} 
Ahn, Minkyu & Guevara Lopez, Miguel Angel & Paternò, Fabio \\
Akbari, Meysam & Guidi, Andrea & Pathan, Risat \\
Al-Majeed, Salah S. & Gulati, Nikhil & Peischl, Bernhard \\
Alonso, Pedro & Guo, Xiaohu & Pereira, Letícia Seixas \\
Althunibat, Saud & Hatzfeld, Christian & Perlin, Hugo Alberto \\
Álvarez García, Juan Antonio & Hayajneh, Khaled F. & Pescapè, Antonio \\
Antoniades, Athos & Hill, Ethan & Petkos, Giorgos \\
Anzt, Hartwig & Hsieh, Shang-Lin & Pontonnier, Charles \\
Atif Tahir, Muhammad & Hu, Chunqiang & Premaladha, J. \\
Ayaz, Hasan & Huang, Di & Prinetto, Paolo \\
Bandodkar, Amay Jairaj & Huang, Po-Chun & Priore, Paolo \\
Barros, Fernando J. & Ibrahim, Zuwairie & Qian, Wei \\
Behzadan, Amir & Jamali, Nawid & Qiao, Yuansong \\
Bogdan, Paul & James, Anne & Qin, Chuan \\
Bojic, Iva & Jamone, Lorenzo & Quang, Pham Tran Anh \\
Bouali, Tarek & Jiménez Naharro, Raúl & Quiniou, Solen \\
Boudjemai, Abdelmadjid & Kandukuri, Somasekhar & Quitadamo, Lucia \\
Burtini, Giuseppe & Kaufmann, Émilie & Radhakrishnan, Chandra \\
Caleffi, Marcello & Kayama, Yoshihiko & Rong, G. \\
Camtepe, Seyit & Kaylani, Hazem & Roque, C. M. C. \\
Cavalin, Paulo R. & Khan, Omar Usman & Salmasi, Nasser \\
Cerny, Tomas & Khan, Wazir Zada & Salomon, Camila P. \\
& & \\
\hline
\end{tabular}




\begin{tabular}{|c|c|c|}
\hline Cha, Hyo Soung & Kheirkhah, Esmaeil & Salvador, Ruben \\
\hline Chambel, Teresa & Kim, Hyunbum & Sánchez, Roberto Therón \\
\hline Chang, Chih-Kai & Kinouchi, Osame & Sanchez Segura, Maribel \\
\hline Chang, Hsien-Tsung & Kleinsmith, Andrea & Schmittner, Christoph \\
\hline Chathuranga, Damith Suresh & Ko, Chun-Hsu & Sharif, Muhammad \\
\hline Chaurasia, Avinash Kumar & Kooi, Thijs & Siever, William \\
\hline Chen, Kai & Kožuh, Ines & Son, Junggab \\
\hline Chen, Xiao-Diao & Kumm, Martin & Song, Seung-Hwan \\
\hline $\mathrm{Chu}$, Shu-Chuan & Lam, Hoang Thanh & Spina, Damiano \\
\hline Congedo, Marco & Le, Nguyen-Thinh & Steinberger, Markus \\
\hline Cukier, Michel & Lee, Hyung-Kew & Taghipour-Gorjikolaie, Mehran \\
\hline Daly, Ian & Leligou, Helen C. (Nelly) & Tagliabue, Jacopo \\
\hline Dantchev, Daniel & Lemma, Saverio & Tamura, Toshiyo \\
\hline Dao, Thi-Kien & Leroy, L. & Tentori, Monica \\
\hline Dewan, M. Ali Akber & Li, Jun-qing & Tiainen, Tarja \\
\hline Doupe, Adam & Li, Tianxing & Tuna, Gurkan \\
\hline Drobics, Mario & Liu, Anfeng & Vardanega, Tullio \\
\hline Erdogmus, Nesli & Liu, Kan & Vazquez-Leal, Hector \\
\hline Erola, Arnau & Liu, Xing & Vegas, Jesús \\
\hline Farooq, Muhammad & Liu, Yanwei & Velas, Martin \\
\hline Feng, Dengguo & Loulergue, Frederic & Vigo, Markel \\
\hline Feng, Jinjuan Heidi & Lozano, Manuel & Walker, Erin \\
\hline Feng, Zhiyong & Ludwig, Simone A. & Wang, Ding \\
\hline Fernandes Jr., Gilberto & Lughofer, Edwin & Wang, Hongwu \\
\hline Fernández Anta, Antonio & Maggi, Sabino & Wang, Ping \\
\hline Findling, Rainhard & Marek-Crnjac, Leila & Weng, Chi-Yao \\
\hline Fredeunburg, Zac & Marino, Francesco & Williams, Patricia A. H. \\
\hline Frommholz, Ingo & Mei, Yi & Winter, Philipp \\
\hline Gadyatskaya, Olga & Milanova, Mariofanna & $\mathrm{Wu}$, Xiaoming \\
\hline García Díaz, Pilar & Mohd Hashim, Siti Zaiton & Zanlungo, Francesco \\
\hline Georgieva, Petia & Montiel, Oscar & Zhang, Liyong \\
\hline Ghobadi, Shahla & Moon, Nammee & Zhang, Yudong \\
\hline Gola, Arkadiusz & Neri, Ferrante & Zhao, Qibin \\
\hline Gomez Soto, Jose Manuel & Nguyen, Trong-The & Zhou, Rigui \\
\hline Gonzalez, Hugo & Ölveczky, Peter Csaba & \\
\hline Graf, Sabine & Ou, Jack & \\
\hline
\end{tabular}

( $) 2017$ by the authors. Submitted for possible open access publication under the terms and conditions of the Creative Commons Attribution (CC-BY) license (http://creativecommons.org/licenses/by/4.0/). 